
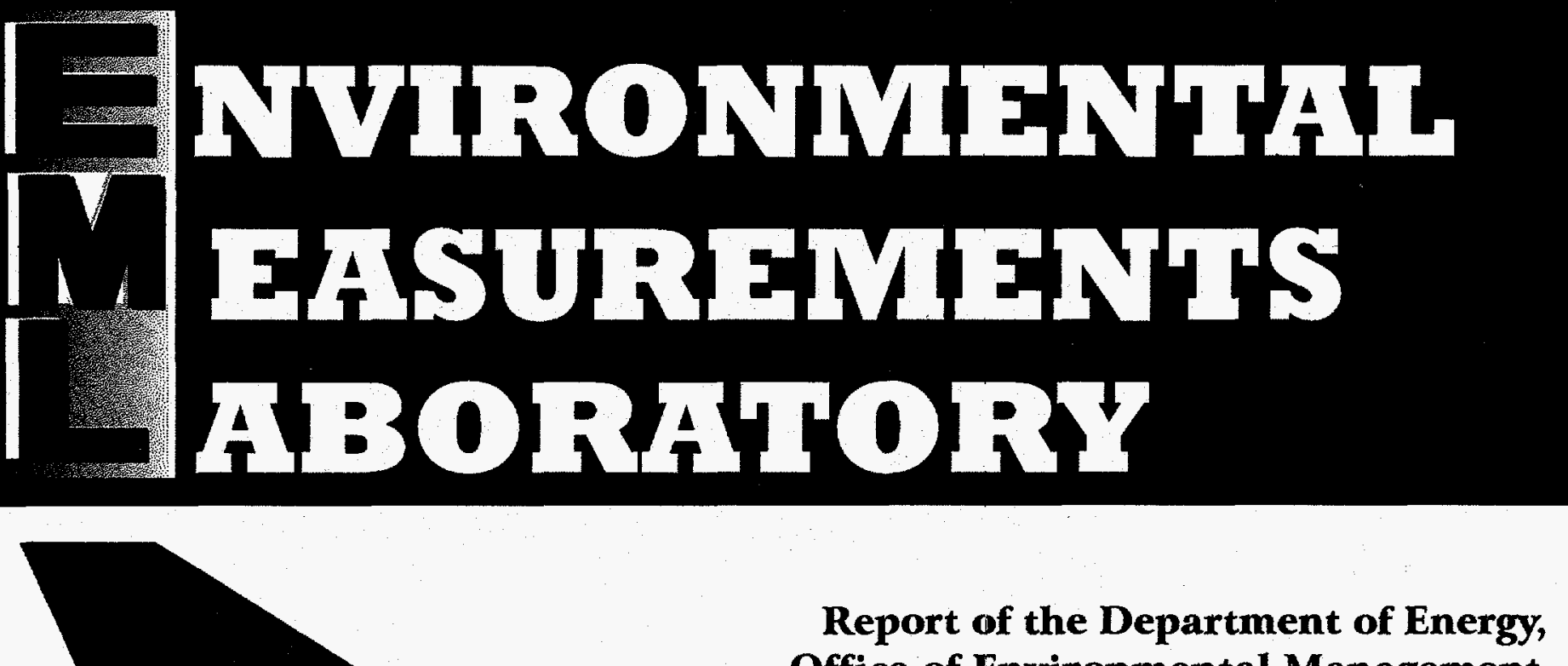

Office of Environmental Management,

Quality Assessment Program, Inorganic Intercomparison Study
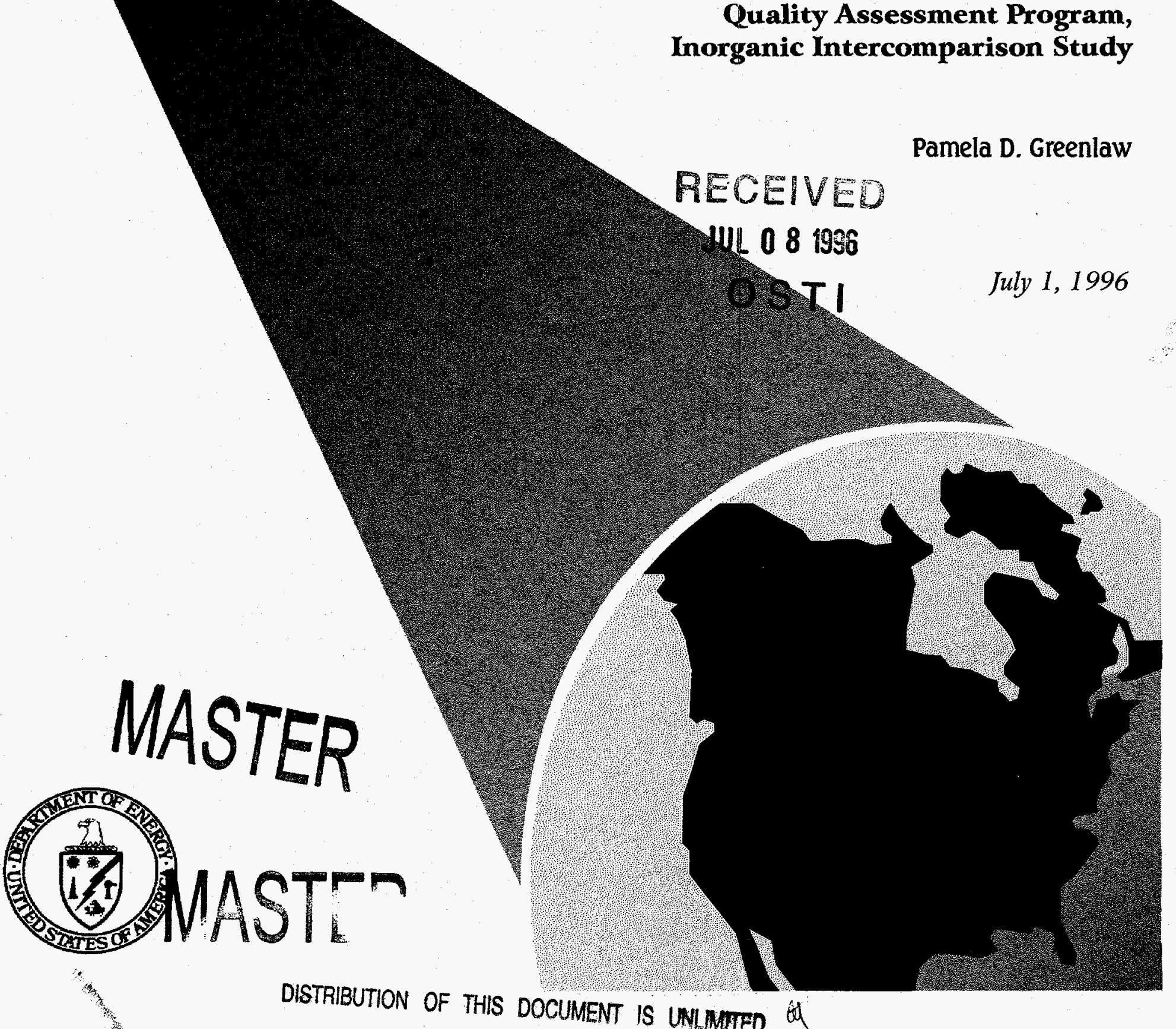
EML-582

\title{
REPORT OF THE DEPARTMENT OF ENERGY, OFFICE OF ENVIRONMENTAL MANAGEMENT, QUALITY ASSESSMENT PROGRAM, INORGANIC INTERCOMPARISON STUDY
}

\author{
Pamela D. Greenlaw \\ Environmental Measurements Laboratory \\ U. S. Department of Energy \\ New York, NY 10014-4811
}

July 1, 1996

\section{DISCLAIMER}

"This report was prepared as an account of work sponsored by an agency of the United States Government. Neither the United States Government nor any agency thereof, nor any of their employees, makes any warranty, express or implied, or assumes any legal liability or responsibility for the accuracy, completeness, or usefulness of any information, apparatus, product, or process disclosed, or represents that its use would not infringe privately owned rights. Reference herein to any specific commercial product, process, or service by trade name, trademark, manufacturer, or otherwise, does not necessarily constitute or imply its endorsement, recommendation, or favoring by the United States Government or any agency thereof. The views and opinions of authors expressed herein do not necessarily state or reflect those of the United States Government or any agency thereof."

This report has been reproduced directly from the best available copy.

Available from the National Technical Information Service, U.S. Department of Commerce, 5285 Port Royal Road, Springfield, Virginia 22161. 


\section{Table of Contents}

QAP 44 Soil Intercomparison Study $\ldots \ldots \ldots \ldots \ldots \ldots \ldots \ldots$ ii

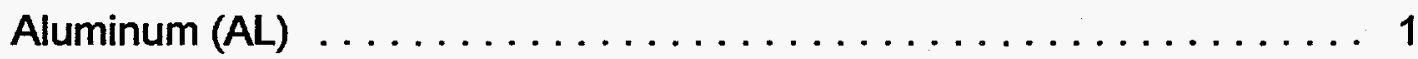

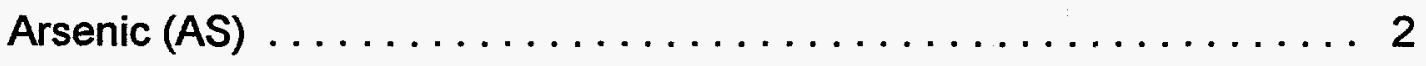

Barium (BA) $\ldots \ldots \ldots \ldots \ldots \ldots \ldots \ldots \ldots \ldots \ldots \ldots \ldots \ldots \ldots \ldots \ldots \ldots$

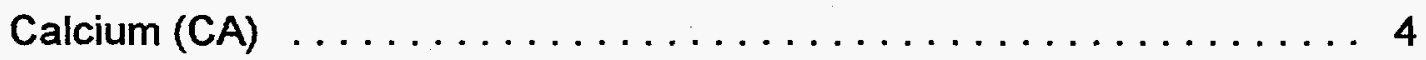

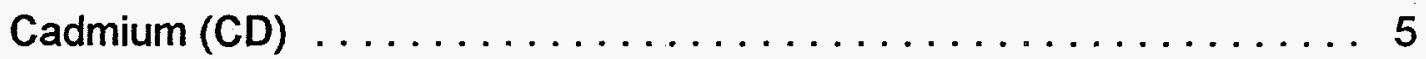

Chromium (CR) $\ldots \ldots \ldots \ldots \ldots \ldots \ldots \ldots \ldots \ldots \ldots \ldots \ldots \ldots \ldots \ldots$

Copper (CU) $\ldots \ldots \ldots \ldots \ldots \ldots \ldots \ldots \ldots \ldots \ldots \ldots \ldots \ldots$

Iron $(\mathrm{FE}) \ldots \ldots \ldots \ldots \ldots \ldots \ldots \ldots \ldots \ldots \ldots \ldots$

Magnesium (MG) $\ldots \ldots \ldots \ldots \ldots \ldots \ldots \ldots \ldots \ldots \ldots$

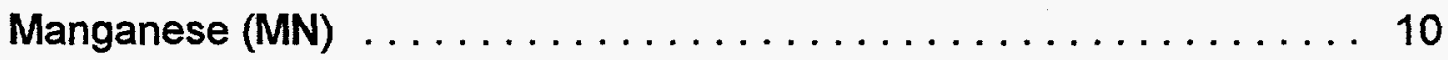

Sodium (NA) $\ldots \ldots \ldots \ldots \ldots \ldots \ldots \ldots \ldots \ldots \ldots \ldots \ldots \ldots \ldots \ldots \ldots \ldots \ldots$

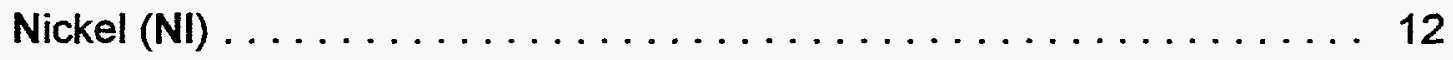

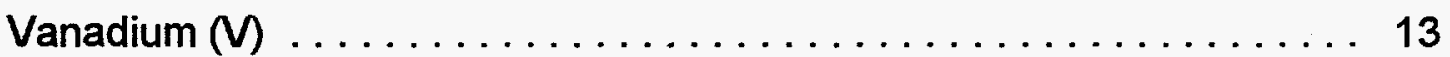

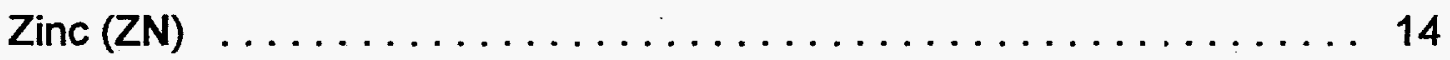

Participating Laboratories $\ldots \ldots \ldots \ldots \ldots \ldots \ldots \ldots \ldots \ldots \ldots$ 


\section{QAP 44 Soil Inorganic Intercomparison Study}

Data for inorganic constituents of the QAP soil sample were collected as part of this distribution. This was a voluntary intercomparison study. The samples were analyzed for RCRA metals using methods $\mathbf{3 0 5 0}$ and 3051 for digestion. Unfortunately the measured values for the elements varied with the digestion method. The value from the $\mathbf{3 0 5 0}$ digestion seemed to be most comparable with the values reported by the other participants so that value was used for the statistical comparison with the participating laboratories. The microwave values are reported as well. We are planning to research the discrepancies between digestion methods and provide more information in the next QAP distribution.

\section{ACKNOWLEGEMENT:}

The US Environmental Protection Agency Region II Technical Support Branch provided confirmatory analysis of the sample for EML. 


\begin{tabular}{|c|c|c|c|c|c|}
\hline Identification & Element & Result & Error & Microwave $\mathrm{HNO} 3$ & Microwave $\mathrm{HNO} / \mathrm{HCl}$ \\
\hline EML & $\overline{\mathrm{AL}}$ & 7085.0 & 235.0 & 12850.0 & 14900.0 \\
\hline 9603SOAG1 & $\mathrm{AL}$ & 7150.0 & 0.0 & & \\
\hline 9603SOAR1 & $\mathrm{AL}$ & 6200.0 & 620.0 & & \\
\hline $9603 \mathrm{SOCH} 1$ & $A L$ & 12200.0 & 1220.0 & & \\
\hline $9603 S O D C 1$ & $\mathrm{AL}$ & 9200.0 & 920.0 & & \\
\hline 9603SOES1 & $\mathrm{AL}$ & 48100.0 & 0.0 & & \\
\hline 9603SOFG1 & $\mathrm{AL}$ & 0.0 & 4600.0 & & \\
\hline 9603SOGA1 & $A L$ & 7580.0 & 0.0 & & \\
\hline 9603SOHL1 & AL & 7100.0 & 0.0 & & \\
\hline 9603SOIS1 & $A L$ & 6940.0 & 0.0 & & \\
\hline 9603SOJP1 & $\mathrm{AL}$ & 41000.0 & 2000.0 & & - \\
\hline 9603SOLA1 & AL & 6424.0 & 206.0 & & \\
\hline 9603SOLA1 & $\mathrm{AL}$ & 8423.0 & 281.0 & & \\
\hline 9603SOLA1 & $\mathrm{AL}$ & 9515.0 & 304.0 & & \\
\hline 9603SOLH1 & $\mathrm{AL}$ & 8360.0 & 0.0 & & \\
\hline 9603SONL1 & AL & 5369.0 & 0.0 & & \\
\hline $96035 O O B 1$ & $A L$ & 6900.0 & 0.0 & & \\
\hline 9603SOOR1 & $\mathrm{AL}$ & 9200.0 & 20.0 & & \\
\hline $9603 S_{0 S C 1}$ & $\mathrm{AL}$ & 7760.0 & 0.0 & & \\
\hline 9603SOSW1 & $\mathrm{AL}$ & 6500.0 & 0.0 & & \\
\hline 9603SOSW1 & $\mathrm{AL}$ & 6560.0 & 0.0 & & \\
\hline 9603SOSW1 & $A L$ & 6700.0 & 0.0 & & \\
\hline 9603SOUC1 & $\mathrm{AL}$ & 10782.0 & 1078.2 & & \\
\hline 9603SOUP1 & $\mathrm{AL}$ & 7560.0 & 0.0 & & \\
\hline
\end{tabular}

Records printed: 24

$\begin{array}{lc}\text { Statistical Summary } \\ \text { (excluding outliers as determined by Kolmogorov-Smirnov Test) } \\ \text { Mean } & 7786 \\ \text { Standard Deviation } & 1638 \\ \text { Median } & 7150 \\ 15 \text { percentile } & 6473 \\ 85 \text { percentile } & 9310 \\ \text { N } & 21\end{array}$

EML results were determined using SW846 method 3050 hotplate digestion with ICP or graphite furnace AA determination. The samples did not appear to totally digest using this method so closed vessel microwave digestion, method 3051 , was used resulting in higher analyte recovery. 


\section{QAP 44 Results by Element}

\begin{tabular}{|c|c|c|c|c|c|}
\hline Identification & Element & Result & Error & Microwave $\mathrm{HNO} 3$ & Microwave $\mathrm{HNO} 3 / \mathrm{HCl}$ \\
\hline EML & AS & 2.4 & 0.1 & 2.3 & \\
\hline 9603SOAG1 & AS & 2.8 & 0.0 & & \\
\hline 9603SOAR1 & AS & 16.0 & 1.6 & & \\
\hline $9603 \mathrm{SOCH} 1$ & AS & 2.4 & 0.2 & & \\
\hline 9603SODC1 & AS & 3.0 & 0.3 & & \\
\hline 9603SOES1 & AS & 5.9 & 0.0 & & \\
\hline 9603SOFG1 & AS & 2.3 & 0.0 & & \\
\hline 9603SOGA1 & AS & 18.5 & 0.0 & & \\
\hline 9603SOHL1 & AS & 1.9 & 0.0 & & \\
\hline 9603SOIS1 & AS & 3.0 & 0.0 & & \\
\hline 9603SOJP1 & AS & 4.0 & 0.2 & & ' \\
\hline 9603SOLA1 & AS & 2.2 & 0.5 & & \\
\hline $9603 S O L A 1$ & AS & 2.5 & 0.5 & & \\
\hline 9603SOLA1 & AS & 2.5 & 1.4 & & \\
\hline 9603SOLH1 & AS & 2.2 & 0.0 & & \\
\hline 9603SONA1 & AS & 3.8 & 0.0 & & \\
\hline 9603SONL1 & AS & 0.0 & 2.0 & & \\
\hline 9603SOOB1 & AS & 2.3 & 0.0 & & \\
\hline 9603SOSC1 & AS & 2.4 & 0.0 & & \\
\hline 9603SOSW1 & AS & 1.5 & 0.0 & & \\
\hline 9603SOSW1 & AS & 1.6 & 0.0 & & \\
\hline 9603SOSW1 & AS & 1.6 & 0.0 & & \\
\hline 9603SOUC1 & AS & 1.3 & 0.1 & & \\
\hline
\end{tabular}

Records printed: 23

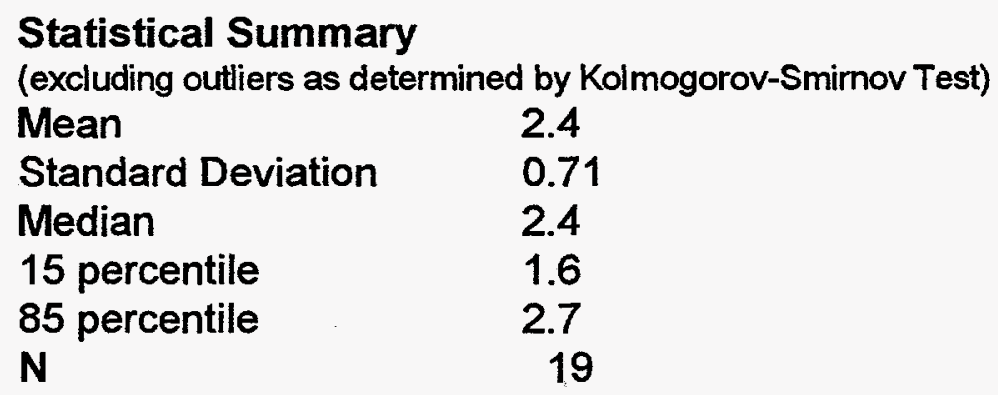

EML results were determined using SW846 method 3050 hotplate digestion with ICP or graphite furnace AA determination. The samples did not appear to totally digest using this method so closed vessel microwave digestion, method 3051, was used resulting in higher analyte recovery. 


\section{QAP 44 Results by Element}

\begin{tabular}{|c|c|c|c|c|c|}
\hline Identification & Element & Result & Error & Microwave $\mathrm{HNO} 3$ & Microwave $\mathrm{HNO} / \mathrm{HCl}$ \\
\hline$\overline{E M L}$ & $\mathrm{BA}$ & 60.5 & 0.5 & 84.5 & 106.5 \\
\hline 9603SOAG1 & BA & 60.7 & 0.0 & & \\
\hline 9603SOAR1 & BA & 54.0 & 5.4 & & \\
\hline $9603 \mathrm{SOCH} 1$ & BA & 69.1 & 6.9 & & \\
\hline 9603SODC1 & BA & 70.0 & 7.0 & & \\
\hline 9603SOES1 & BA & 229.0 & 0.0 & & \\
\hline 9603SOFG1 & BA & 46.0 & 0.0 & & \\
\hline 9603SOGA1 & BA & 59.5 & 0.0 & & \\
\hline 9603SOHL1 & BA & 60.0 & 0.0 & & \\
\hline 9603SOIS1 & BA & 62.8 & 0.0 & & \\
\hline 9603SOJP1 & BA & 190.0 & 0.0 & & - \\
\hline 9603SOLA1 & BA & 56.0 & 7.0 & & \\
\hline 9603SOLA1 & BA & 66.2 & 8.3 & & \\
\hline 9603SOLA1 & BA & 72.0 & 2.0 & & \\
\hline 9603SOLH1 & BA & 67.5 & 0.0 & & \\
\hline 9603SONA1 & BA & 82.9 & 0.0 & & \\
\hline 9603SONL1 & BA & 51.7 & 0.0 & & \\
\hline 9603SOOB1 & BA. & 60.0 & 0.0 & & \\
\hline 9603SOOR1 & BA & 71.1 & 0.3 & & \\
\hline 9603SOSC1 & BA & 63.1 & 0.0 & & \\
\hline 9603SOSW1 & BA & 59.2 & 0.0 & & \\
\hline 9603SOSW1 & BA & 59.6 & 0.0 & & \\
\hline 9603SOSW1 & BA & 60.7 & 0.0 & & \\
\hline 9603SOUC1 & BA & 73.4 & 7.3 & & \\
\hline 9603SOUP1 & BA & 66.3 & 0.0 & & \\
\hline
\end{tabular}

Records printed: 25

$\begin{array}{ll}\text { Statistical Summary } \\ \text { (excluding outliers as determined by Kolmogorov-Smirnov Test) } \\ \text { Mean } & 63.1 \\ \text { Standard Deviation } & 8.0 \\ \text { Median } & 60.7 \\ \text { 15 percentile } & 55.9 \\ 85 \text { percentile } & 71.1 \\ \text { N } & 23\end{array}$

EML results were determined using SW846 method 3050 hotplate digestion with ICP or graphite furnace AA determination. The samples did not appear to totally digest using this method so closed vessel microwave digestion, method 3051, was used resulting in higher analyte recovery. 


\section{QAP 44 Results by Element}

\begin{tabular}{|c|c|c|c|c|c|}
\hline Identification & Element & Result & Error & Microwave $\mathrm{HNO} 3$ & Microwave $\mathrm{HNO} 3 / \mathrm{HCl}$ \\
\hline EML & $\overline{C A}$ & 4050.0 & 30.0 & 3870.0 & 4190.0 \\
\hline 9603SOAG1 & $C A$ & 4480.0 & 0.0 & & \\
\hline 9603SOAR1 & CA & 3900.0 & 390.0 & & \\
\hline $9603 \mathrm{SOCH} 1$ & $C A$ & 4650.0 & 465.0 & & \\
\hline 9603SODC1 & CA & 4400.0 & 440.0 & & \\
\hline 9603SOES1 & $\mathrm{CA}$ & 6100.0 & 0.0 & & \\
\hline 9603 SOFG1 & CA & 4400.0 & 0.0 & & \\
\hline 9603SOGA1 & CA & 3850.0 & 0.0 & & \\
\hline 9603SOHL1 & CA & 4200.0 & 0.0 & & \\
\hline 9603SOIS1 & CA & 4640.0 & 0.0 & & \\
\hline 9603SOJP1 & $\mathrm{CA}$ & 5700.0 & 870.0 & & - \\
\hline 9603SOLA1 & CA & 3871.0 & 248.0 & & \\
\hline 9603SOLA1 & CA & 4115.0 & 118.0 & & \\
\hline 9603SOLA1 & CA & 4187.0 & 174.0 & & \\
\hline 9603SOLH1 & CA & 4670.0 & 0.0 & & \\
\hline 9603SONL1 & CA & 3932.0 & 0.0 & & \\
\hline $960350 O B 1$ & CA & 4000.0 & 0.0 & & \\
\hline 9603SOOR1 & CA & 4730.0 & 50.0 & & \\
\hline $9603 \mathrm{SOSC} 1$ & $\mathrm{CA}$ & 3950.0 & 0.0 & & \\
\hline 9603SOSW1 & CA & 4430.0 & 0.0 & & \\
\hline 9603SOSW1 & CA & 4450.0 & 0.0 & & \\
\hline 9603SOSW1 & CA & 4470.0 & 0.0 & & \\
\hline 9603SOUC1 & $\mathrm{CA}$ & 4073.0 & 407.0 & & \\
\hline 9603SOUP1 & $\mathrm{CA}$ & 4710.0 & 0.0 & & \\
\hline
\end{tabular}

Records printed: 24

$\begin{array}{lc}\text { Statistical Summary } \\ \text { (excluding outliers as determined by Kolmogorov-Smirnov Test) } \\ \text { Mean } & 4280 \\ \text { Standard Deviation } & 301 \\ \text { Median } & 4300 \\ 15 \text { percentile } & 3926 \\ 85 \text { percentile } & 4654 \\ \text { N } & 22\end{array}$

EML results were determined using SW846 method 3050 hotplate digestion with ICP or graphite furnace AA determination. The samples did not appear to totally digest using this method so closed vessel microwave digestion, method 3051, was used resulting in higher analyte recovery. 


\section{QAP 44 Results by Element}

\begin{tabular}{|c|c|c|c|c|c|}
\hline Identification & Element & Result & Error & Microwave $\mathrm{HNO} 3$ & Microwave $\mathrm{HNO} 3 / \mathrm{HCl}$ \\
\hline$\overline{E M L}$ & $\overline{C D}$ & $<1$ & & $<1$ & $<1$ \\
\hline 9603SOAG1 & $C D$ & 0.29 & 0.0 & & \\
\hline 9603SOAR1 & $C D$ & 0 & 0.5 & & \\
\hline $9603 \mathrm{SOCH} 1$ & $C D$ & 0.218 & & & \\
\hline 9603SODC1 & $C D$ & 0.13 & 0.0 & & \\
\hline 9603SOES1 & $C D$ & 0.083 & & & \\
\hline 9603SOFG1 & $C D$ & 0 & 0.0 & & \\
\hline 9603SOGA1 & $C D$ & 1.8 & 0.0 & & \\
\hline 9603SOHL1 & $C D$ & 0.03 & & & \\
\hline 9603SOIS1 & $C D$ & 0 & 0.0 & & \\
\hline 9603SOJP1 & $C D$ & 7 & 0.0 & & . \\
\hline 9603SOLA1 & $C D$ & 0.256 & & & \\
\hline 9603SOLA1 & $C D$ & 0.279 & 0.1 & & \\
\hline 9603SOLA1 & $C D$ & 0.492 & 0.1 & & \\
\hline 9603SOLH1 & $C D$ & 0.23 & 0.0 & & \\
\hline 9603SONL1 & $C D$ & 0 & 1.0 & & \\
\hline $9603 S O O B 1$ & $C D$ & 0 & & & \\
\hline 9603SOOR1 & $C D$ & 0.889 & 0.2 & & \\
\hline $9603 S_{0 S C} 1$ & $C D$ & 0 & 0.0 & & \\
\hline 9603SOSW1 & $C D$ & 0.072 & 0.0 & & \\
\hline
\end{tabular}

Records printed: 20

$\begin{array}{ll}\text { Statistical Summary } \\ \text { (excluding outliers as determined by Kolmogorov-Smirnov Test) } \\ \text { Mean } & 0.22 \\ \text { Standard Deviation } & 0.13 \\ \text { Median } & 0.23 \\ 15 \text { percentile } & 0.074 \\ 85 \text { percentile } & 0.31 \\ \text { N } & 10\end{array}$

EML results were determined using SW846 method 3050 hotplate digestion with ICP or graphite furnace AA determination. The samples did not appear to totally digest using this method so closed vessel microwave digestion, method 3051 , was used resulting in higher analyte recovery. 


\section{QAP 44 Results by Element}

\begin{tabular}{|c|c|c|c|c|c|}
\hline$\frac{\text { Identification }}{E M L}$ & $\frac{\text { Element }}{\mathrm{CR}}$ & $\frac{\text { Result }}{12.5}$ & $\frac{\text { Error }}{0.5}$ & $\frac{\text { Microwave HNO3 }}{18.5}$ & $\frac{\text { Microwave } \mathrm{HNO} 3 / \mathrm{HCl}}{21.5}$ \\
\hline 9603SOAG1 & CR & 13.9 & 0.0 & & \\
\hline 9603SOAR1 & CR & 15.0 & 1.5 & & \\
\hline $9603 S O C H 1$ & $\mathrm{CR}$ & 15.9 & 1.6 & & \\
\hline 9603SODC1 & $\mathrm{CR}$ & 19.0 & 1.9 & & \\
\hline 9603SOES1 & $\mathrm{CR}$ & 51.5 & 0.0 & & \\
\hline 9603SOFG1 & CR & 9.3 & 0.0 & & \\
\hline 9603SOGA1 & CR & 15.4 & 0.0 & & \\
\hline 9603SOHL1 & CR & 17.0 & 0.0 & & \\
\hline 9603SOIS1 & CR & 12.9 & 0.0 & & \\
\hline 9603SOJP1 & CR & 34.0 & 1.0 & & ${ }^{\circ}$ \\
\hline 9603SOLA1 & CR & 13.2 & 2.1 & & \\
\hline 9603SOLA1 & CR & 15.2 & 2.4 & & \\
\hline 9603SOLA1 & CR & 17.9 & 6.9 & & \\
\hline 9603SOLH1 & $\mathrm{CR}$ & 16.2 & 0.0 & & - \\
\hline 9603SONA1 & CR & 22.2 & 0.0 & & \\
\hline 9603SONL1 & CR & 12.7 & 0.0 & & \\
\hline $9603500 B 1$ & $\mathrm{CR}$ & 13.0 & 0.0 & & \\
\hline 9603SOOR1 & CR & 17.0 & 0.2 & & \\
\hline 9603SOSC1 & CR & 16.3 & 0.0 & & \\
\hline 9603SOSW1 & CR & 11.4 & 0.0 & & \\
\hline 9603SOSW1 & $\mathrm{CR}$ & 11.6 & 0.0 & & \\
\hline 9603SOSW1 & CR & 11.8 & 0.0 & & \\
\hline 9603SOUC1 & $\mathrm{CR}$ & 31.0 & 3.1 & & \\
\hline 9603SOUP1 & CR & 14.5 & 0.0 & & \\
\hline
\end{tabular}

Records printed: 25

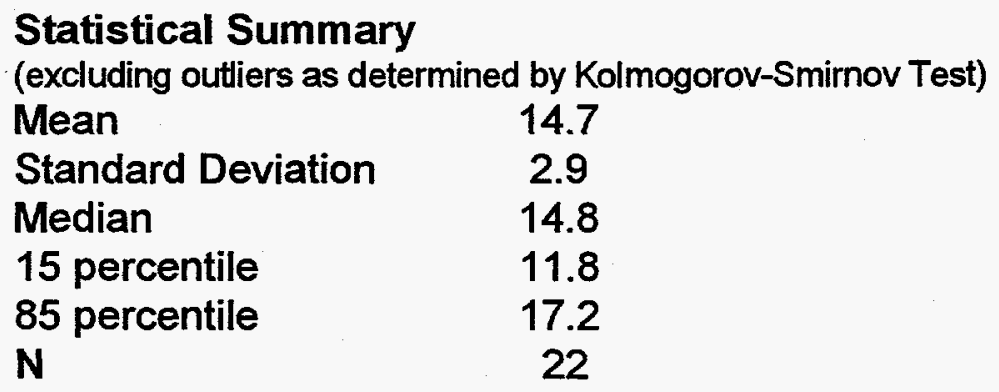

EML results were determined using SW846 method 3050 hotplate digestion with ICP or graphite furnace AA determination. The samples did not appear to totally digest using this method so closed vessel microwave digestion, method 3051 , was used resulting in higher analyte recovery. 


\section{QAP 44 Results by Element}

\begin{tabular}{|c|c|c|c|c|c|}
\hline Identification & Element & Result & Error & Microwave $\mathrm{HNO} 3$ & Microwave $\mathrm{HNO} 3 / \mathrm{HCl}$ \\
\hline$\overline{E M L}$ & $\mathrm{CU}$ & 9.5 & 1.5 & 10.5 & 11.0 \\
\hline 9603SOAG1 & CU & 8.8 & 0.0 & & \\
\hline 9603SOAR1 & $\mathrm{CU}$ & 9.0 & 0.9 & & \\
\hline $9603 \mathrm{SOCH} 1$ & CU & 14.9 & 1.5 & & \\
\hline 9603SODC1 & $\mathrm{CU}$ & 11.0 & 1.1 & & \\
\hline 9603SOES1 & $\mathrm{CU}$ & 7.1 & 0.0 & & \\
\hline 9603 SOFG1 & CU & 6.4 & 0.0 & & \\
\hline 9603SOGA1 & $\mathrm{CU}$ & 8.0 & 0.0 & & \\
\hline 9603SOHL1 & $\mathrm{CU}$ & 8.6 & 0.0 & & \\
\hline 9603SOIS1 & $\mathrm{CU}$ & 8.0 & 0.0 & & \\
\hline 9603SOJP1 & $\mathrm{CU}$ & 300.0 & 0.0 & & \\
\hline 9603SOLA1 & CU & 6.9 & 0.5 & & \\
\hline 9603SOLA1 & $\mathrm{CU}$ & 7.6 & 0.5 & & \\
\hline 9603SOLA1 & $\mathrm{CU}$ & 7.9 & 0.6 & & \\
\hline 9603SONL1 & CU & 6.0 & 0.0 & & \\
\hline $960350 O B 1$ & $\mathrm{CU}$ & 8.0 & 0.0 & & \\
\hline 9603SOOR1 & CU & 10.0 & 0.3 & & \\
\hline 9603SOSC1 & $\mathrm{CU}$ & 8.3 & 0.0 & & \\
\hline 9603SOSW1 & $\mathrm{CU}$ & 8.1 & 0.0 & & \\
\hline 9603SOSW1 & $\mathrm{CU}$ & 8.3 & 0.0 & & \\
\hline 9603SOSW1 & $\mathrm{CU}$ & 8.4 & 0.0 & & \\
\hline 9603SOUC1 & $\mathrm{CU}$ & 10.7 & 1.1 & & \\
\hline 9603SOUP1 & CU & 9.4 & ח & & \\
\hline
\end{tabular}

Records printed: 23

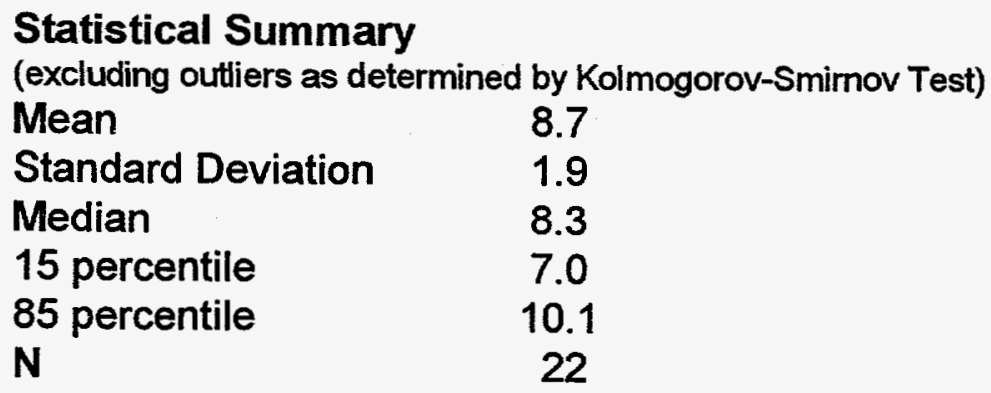

EML results were determined using SW846 method 3050 hotplate digestion with ICP or graphite furnace AA determination. The samples did not appear to totally digest using this method so closed vessel microwave digestion, method 3051 , was used resulting in higher analyte recovery. 


\section{QAP 44 Results by Element}

\begin{tabular}{|c|c|c|c|c|c|}
\hline Identification & Element & Result & Error & Microwave $\mathrm{HNO} 3$ & Microwave $\mathrm{HNO} 3 / \mathrm{HCl}$ \\
\hline$\overline{E M L}$ & $\overline{\mathrm{FE}}$ & $1 \overline{1450.0}$ & $\overline{250.0}$ & 14400.0 & 12750.0 \\
\hline 9603SOAG1 & FE & 13600.0 & 0.0 & & \\
\hline 9603SOAR1 & $\mathrm{FE}$ & 10000.0 & 1000.0 & & \\
\hline $9603 \mathrm{SOCH} 1$ & FE & 14200.0 & 1420.0 & & \\
\hline 9603SODC1 & FE & 11900.0 & 0.0 & & \\
\hline 9603SOES1 & FE & 13700.0 & 0.0 & & \\
\hline 9603SOFG1 & FE & 8100.0 & 0.0 & & \\
\hline 9603SOGA1 & FE & 10100.0 & 0.0 & & \\
\hline 9603SOHL1 & FE & 12000.0 & 0.0 & & \\
\hline 9603SOIS1 & $\mathrm{FE}$ & 11900.0 & 0.0 & & \\
\hline 9603SOJP1 & $\mathrm{FE}$ & 15000.0 & 300.0 & & - \\
\hline 9603SOLA1 & FE & 10238.0 & 362.0 & & \\
\hline 9603SOLA1 & FE & 11776.0 & 365.0 & & \\
\hline 9603SOLA1 & FE & 12320.0 & 380.0 & & \\
\hline 9603SOLH1 & $\mathrm{FE}$ & 12600.0 & 0.0 & & \\
\hline 9603SONL1 & $\mathrm{FE}$ & 10750.0 & 0.0 & & \\
\hline 9603SOOB1 & FE & 12000.0 & 0.0 & & \\
\hline 9603SOOR1 & FE & 13010.0 & 10.0 & & \\
\hline 9603SOSC1 & FE & 11500.0 & 0.0 & & \\
\hline 9603SOSW1 & FE & 10800.0 & 0.0 & & \\
\hline 9603SOSW1 & FE & 11000.0 & 0.0 & & \\
\hline 9603SOUC1 & FE & 13074.0 & 1307.4 & & \\
\hline 9603SOUP1 & 18 & 11900.0 & 0.0 & & \\
\hline
\end{tabular}

Records printed: 23

$\begin{array}{lc}\text { Statistical Summary } \\ \text { (excluding outliers as determined by Kolmogorov-Smirnov Test) } \\ \text { Mean } & 11866 \\ \text { Standard Deviation } & 1538 \\ \text { Median } & 11900 \\ \text { 15 percentile } & 10231 \\ 85 \text { percentile } & 13605 \\ \text { N } & 23\end{array}$

EML results were determined using SW846 method 3050 hotplate digestion with ICP or graphite furnace AA determination. The samples did not appear to totally digest using this method so closed vessel microwave digestion, method 3051, was used resulting in higher analyte recovery. 


\section{QAP 44 Results by Element}

\begin{tabular}{|c|c|c|c|c|c|}
\hline Identification & Element & Result & Error & Microwave HNO3 & Microwave $\mathrm{HNO} 3 / \mathrm{HCl}$ \\
\hline EML & $\overline{M G}$ & $\overline{2845.0}$ & 5.0 & 3095.0 & 3410.0 \\
\hline $9603 S O A G 1$ & MG & 2990.0 & 0.0 & & \\
\hline 9603SOAR1 & MG & 2600.0 & 260.0 & & \\
\hline $9603 \mathrm{SOCH} 1$ & MG & 3470.0 & 347.0 & & \\
\hline $9603 S O D C 1$ & MG & 380.0 & 38.0 & & \\
\hline 9603SOES1 & MG & 2140.0 & 0.0 & & \\
\hline 9603SOFG1 & MG & 2300.0 & 0.0 & & \\
\hline 9603SOGA1 & MG & 2790.0 & 0.0 & & \\
\hline 9603SOHL1 & MG & 3200.0 & 0.0 & & \\
\hline 9603SOIS1 & MG & 3120.0 & 0.0 & & \\
\hline 9603SOJP1 & MG & 12000.0 & 1100.0 & & \\
\hline 9603SOLA1 & MG & 2780.0 & 50.0 & & \\
\hline 9603SOLA1 & MG & 3005.0 & 54.0 & & \\
\hline 9603SOLA1 & MG & 3116.0 & 56.0 & & \\
\hline 9603SOLH1 & MG & 3290.0 & 0.0 & & \\
\hline 9603SONL1 & MG & 2528.0 & 0.0 & & \\
\hline 9603SOOB1 & MG & 2700.0 & 0.0 & & \\
\hline 9603SOOR1 & MG & 3250.0 & 10.0 & & \\
\hline 9603 SOSC1 & MG & 2940.0 & 0.0 & & \\
\hline 9603SOSW1 & MG & 2830.0 & 0.0 & & \\
\hline 9603SOSW1 & MG & 2850.0 & 0.0 & & \\
\hline 9603SOUC1 & MG & 3325.0 & 333.0 & & \\
\hline 9603SOUP1 & MG & 3290.0 & 0.0 & & \\
\hline
\end{tabular}

Records printed: 23

$\begin{array}{lc}\text { Statistical Summary } \\ \text { (excluding outliers as determined by Kolmogorov-Smirnov Test) } \\ \text { Mean } & 2922 \\ \text { Standard Deviation } & 344 \\ \text { Median } & 2940 \\ 15 \text { percentile } & 2574 \\ 85 \text { percentile } & 3290 \\ \text { N } & 21\end{array}$

EML results were determined using SW846 method 3050 hotplate digestion with ICP or graphite furnace AA determination. The samples did not appear to totally digest using this method so closed vessel microwave digestion, method 3051, was used resulting in higher analyte recovery. 


\section{QAP 44 Results by Element}

\begin{tabular}{|c|c|c|c|c|c|}
\hline Identification & Element & $\underline{\text { Result }}$ & Error & Microwave $\mathrm{HNO} 3$ & Microwave $\mathrm{HNO} / \mathrm{HCl}$ \\
\hline EML & $\mathrm{MN}$ & 355.5 & 0.5 & 351.0 & 372.0 \\
\hline 9603SOAG1 & $M N$ & 380.0 & 0.0 & & \\
\hline 9603SOAR1 & MN & 340.0 & 34.0 & & \\
\hline $9603 \mathrm{SOCH} 1$ & MN & 390.0 & 39.0 & & \\
\hline 9603SODC1 & MN & 3100.0 & 310.0 & & \\
\hline 9603SOES1 & MN & 401.0 & 0.0 & & \\
\hline 9603SOFG1 & MN & 280.0 & 0.0 & & \\
\hline 9603SOGA1 & MN & 338.0 & 0.0 & & \\
\hline 9603SOHL1 & MN & 380.0 & 0.0 & & \\
\hline 9603SOIS1 & $M N$ & 370.0 & 0.0 & & \\
\hline 9603SOJP1 & $M N$ & 430.0 & 8.0 & & ' \\
\hline 9603SOLA1 & MN & 317.0 & 16.0 & & \\
\hline 9603SOLA1 & MN & 338.0 & 17.0 & & \\
\hline 9603SOLA1 & MN & 350.0 & 20.0 & & \\
\hline 9603SOLH1 & MN & 366.0 & 0.0 & & \\
\hline 9603SONL1 & MN & 342.0 & 0.0 & & \\
\hline $9603 S O O B 1$ & MN & 350.0 & 0.0 & & \\
\hline 9603SOOR1 & $M N$ & 372.0 & 1.0 & & \\
\hline 9603SOSC1 & MN & 353.0 & 0.0 & & \\
\hline 9603SOSW1 & MN & 369.0 & 0.0 & & \\
\hline 9603SOSW1 & MN & 371.0 & 0.0 & & \\
\hline 9603SOSW1 & $M N$ & 372.0 & 0.0 & & \\
\hline 9603SOUC1 & MN & 401.0 & 40.1 & & \\
\hline 9603SOUP1 & MN & 406.0 & 0.0 & & \\
\hline
\end{tabular}

Records printed: 24

$\begin{array}{lc}\text { Statistical Summary } \\ \text { (excluding outliers as determined by Kolmogorov-Smirnov Test) } \\ \text { Mean } & 363 \\ \text { Standard Deviation } & 32 \\ \text { Median } & 369 \\ \text { 15 percentile } & 338 \\ 85 \text { percentile } & 401 \\ \text { N } & 23\end{array}$

EML results were determined using SW846 method 3050 hotplate digestion with ICP or graphite furnace AA determination. The samples did not appear to totally digest using this method so closed vessel microwave digestion, method 3051 , was used resulting in higher analyte recovery. 


\section{QAP 44 Results by Element}

\begin{tabular}{|c|c|c|c|c|c|}
\hline$\frac{\text { Identification }}{E M L}$ & $\frac{\text { Element }}{\mathrm{NA}}$ & $\frac{\text { Result }}{5500}$ & Error & Microwave $\mathrm{HNO} 3$ & $\frac{\text { Microwave HNO3/HCl }}{<500}$ \\
\hline $\begin{array}{l}\text { EML } \\
\text { 9603SOAG1 }\end{array}$ & NA & 234 & 0.0 & & \\
\hline 9603SOAR1 & NA & 290 & 29.0 & & \\
\hline $9603 \mathrm{SOCH} 1$ & NA & 450 & 45.0 & & \\
\hline 9603SODC1 & NA & 340 & 34.0 & & \\
\hline 9603SOES1 & NA & 5040 & 0.0 & & \\
\hline $96035 O F G 1$ & NA & 170 & 0.0 & & \\
\hline 9603SOGA1 & NA & 301 & 0.0 & & \\
\hline 9603SOHL1 & NA & 380 & 0.0 & & \\
\hline 9603SOIS1 & NA & 283 & 0.0 & & \\
\hline 9603SOJP1 & NA & 7100 & 50.0 & & \\
\hline 9603SOLA1 & NA & 273 & 32.0 & & \\
\hline 9603SOLA1 & NA & 311 & 37.0 & & \\
\hline 9603SOLA1 & NA & 348 & 41.0 & & \\
\hline 9603SONL1 & NA & 0 & 2000.0 & & \\
\hline $9603 S O O B 1$ & NA & 400 & 0.0 & & \\
\hline 9603SOOR1 & NA & 283 & 1.0 & & \\
\hline $9603 \mathrm{SOSC} 1$ & NA & 324 & 0.0 & & \\
\hline 9603SOSW1 & NA & 247 & 0.0 & & \\
\hline 9603SOSW1 & NA & 252 & 0.0 & & \\
\hline 9603SOSW1 & NA & 255 & 0.0 & & \\
\hline 9603SOUC1 & NA & 500 & 50.0 & & \\
\hline 9603SOUP1 & NA & 300 & 0.0 & & \\
\hline
\end{tabular}

Records printed: 23

\begin{tabular}{|c|c|}
\hline $\begin{array}{l}\text { Statistical Summa } \\
\text { (excluding outliers as d }\end{array}$ & \\
\hline Mean & 313 \\
\hline Standard Deviation & 76 \\
\hline Median & 300 \\
\hline 15 percentile & 250 \\
\hline 85 percentile & 390 \\
\hline $\mathbf{N}$ & 19 \\
\hline
\end{tabular}

EML results were determined using SW846 method 3050 hotplate digestion with ICP or graphite furnace AA determination. The samples did not appear to totally digest using this method so closed vessel microwave digestion, method 3051, was used resulting in higher analyte recovery. 


\section{QAP 44 Results by Element}

\begin{tabular}{|c|c|c|c|c|c|}
\hline Identification & Element & Result & Error & Microwave $\mathrm{HNO} 3$ & Microwave $\mathrm{HNO} 3 / \mathrm{HCl}$ \\
\hline EML & & 24.5 & 0.5 & 26.0 & 26.0 \\
\hline 9603SOAG1 & NI & 25.5 & 0.0 & & \\
\hline 9603SOAR1 & $\mathrm{NI}$ & 24.0 & 2.4 & & \\
\hline 9603SOCH1 & $\mathrm{NI}$ & 27.4 & 2.7 & & \\
\hline 9603SODC1 & $\mathrm{NI}$ & 27.0 & 2.7 & & \\
\hline 9603SOFG1 & $\mathrm{NI}$ & 20.0 & 0.0 & & \\
\hline 9603SOGA1 & $\mathrm{NI}$ & 25.2 & 0.0 & & \\
\hline 9603SOHL1 & $\mathrm{NI}$ & 28.0 & 0.0 & & \\
\hline 9603SOIS1 & $\mathrm{NI}$ & 25.7 & 0.0 & & \\
\hline 9603SOJP1 & $\mathrm{NI}$ & 60.0 & 0.0 & & \\
\hline 9603SOLA1 & $\mathrm{NI}$ & 34.4 & 2.3 & & - \\
\hline 9603SOLA1 & $\mathrm{NI}$ & 35.0 & 2.0 & & \\
\hline 9603SOLA1 & $\mathrm{NI}$ & 38.9 & 2.6 & & \\
\hline 9603SOLH1 & NI & 28.9 & 0.0 & & \\
\hline 9603SONL1 & $\mathrm{NI}$ & 23.8 & 0.0 & & \\
\hline 9603SOOB1 & $\mathrm{NI}$ & 24.0 & 0.0 & & \\
\hline 9603SOOR1 & $\mathrm{NI}$ & 26.3 & 1.1 & & \\
\hline 9603SOSC1 & $\mathrm{NI}$ & 25.7 & 0.0 & & \\
\hline 9603SOSW1 & $\mathrm{NI}$ & 25.4 & 0.0 & & \\
\hline 9603SOSW1 & NI & 25.5 & 0.0 & & \\
\hline 9603SOSW1 & $\mathrm{NI}$ & 26.1 & 0.0 & & \\
\hline 9603SOUC1 & $\mathrm{NI}$ & 45.2 & 4.5 & & \\
\hline 9603SOUP1 & $\mathrm{NI}$ & 28.3 & 0.0 & & \\
\hline
\end{tabular}

Records printed: 23

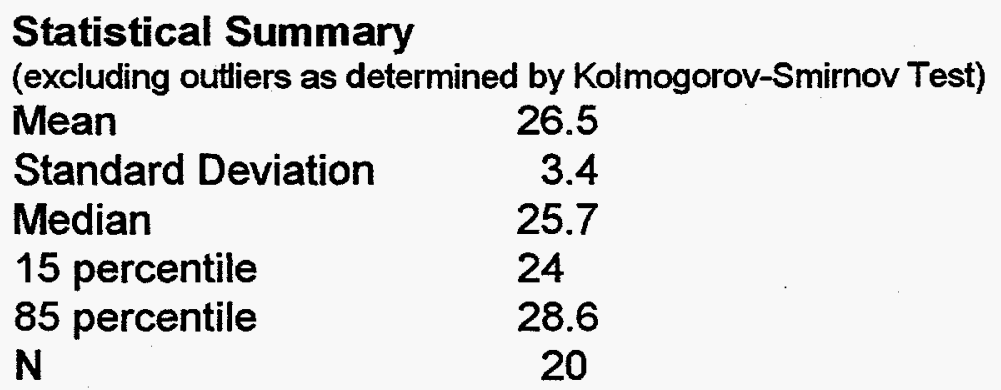

EML results were determined using SW846 method 3050 hotplate digestion with ICP or graphite furnace AA determination. The samples did not appear to totally digest using this method so closed vessel microwave digestion, method 3051, was used resulting in higher analyte recovery. 


\section{QAP 44 Results by Element}

\begin{tabular}{llrrrr} 
Identification & Element & Result & Error & Microwave HNO3 & Microwave HNO3/HCl \\
\cline { 1 - 2 } EML & $V$ & 8.0 & 0.0 & 14.5 & 18.5 \\
9603SOAG1 & $V$ & 9.0 & 0.0 & & \\
9603SOAR1 & $V$ & 7.2 & 0.7 & & \\
9603SOCH1 & $V$ & 10.2 & 1.0 & \\
9603SODC1 & $V$ & 11.0 & 1.1 & \\
9603SOES1 & $V$ & 19.0 & 0.0 & \\
9603SOFG1 & $V$ & 5.8 & 0.0 & \\
9603SOGA1 & $V$ & 10.5 & 0.0 & \\
9603SOHL1 & $V$ & 9.3 & 0.0 & \\
9603SOIS1 & $V$ & 6.5 & 0.0 & \\
9603SOJP1 & $V$ & 34.0 & 2.9 & \\
9603SOLA1 & $V$ & 6.8 & 1.5 & \\
9603SOLA1 & $V$ & 9.7 & 2.1 & \\
9603SOLA1 & $V$ & 10.4 & 0.6 & \\
9603SOLH1 & $V$ & 10.0 & 0.0 & \\
9603SONL1 & $V$ & 0.0 & 10.0 & \\
9603SOOB1 & $V$ & 8.0 & 0.0 & \\
9603SOOR1 & $V$ & 10.7 & 0.2 & \\
9603SOSC1 & $V$ & 9.1 & 0.0 & \\
9603SOSW1 & $V$ & 7.9 & 0.0 & \\
9603SOSW1 & $V$ & 8.6 & 0.0 & \\
9603SOSW1 & $V$ & 8.9 & 0.0 & \\
9603SOUC1 & $V$ & 34.4 & 3.4 & \\
9603SOUP1 & $V$ & 9.3 & 0.0 &
\end{tabular}

Records printed: 24

\begin{tabular}{|c|c|}
\hline $\begin{array}{l}\text { Statistical Summ } \\
\text { (excluding outliers as d }\end{array}$ & d by Kolmogorov-Smirnov Test \\
\hline Mean & 8.8 \\
\hline Standard Deviation & 1.4 \\
\hline Median & 9.1 \\
\hline 15 percentile & 7.0 \\
\hline 85 percentile & 10.4 \\
\hline $\mathbf{N}$ & 20 \\
\hline
\end{tabular}

EML results were determined using SW846 method 3050 hotplate digestion with ICP or graphite furnace AA determination. The samples did not appear to totally digest using this method so closed vessel microwave digestion, method 3051, was used resulting in higher analyte recovery. 


\section{QAP 44 Results by Element}

\begin{tabular}{|c|c|c|c|c|c|}
\hline Identification & Element & Result & Error & Microwave $\mathrm{HNO} 3$ & Microwave $\mathrm{HNO} / \mathrm{HCl}$ \\
\hline$\overline{E M L}$ & $\overline{\mathrm{ZN}}$ & 33.0 & 0.0 & 35.5 & 35.0 \\
\hline 9603SOAG1 & $\mathrm{ZN}$ & 35.2 & 0.0 & & \\
\hline 9603SOAR1 & $\mathrm{ZN}$ & 33.0 & 3.3 & & \\
\hline $9603 \mathrm{SOCH} 1$ & ZN & 44.0 & 4.4 & & \\
\hline 9603SODC1 & $\mathrm{ZN}$ & 35.0 & 3.5 & & \\
\hline 9603 SOES1 & ZN & 32.7 & 0.0 & & \\
\hline 9603SOFG1 & $\mathrm{ZN}$ & 25.0 & 0.0 & & \\
\hline 9603SOGA1 & $\mathrm{ZN}$ & 33.7 & 0.0 & & \\
\hline 9603SOHL1 & ZN & 38.0 & 0.0 & & \\
\hline 9603SOIS1 & $\mathrm{ZN}$ & 34.0 & 0.0 & & \\
\hline 9603SOJP1 & $\mathrm{ZN}$ & 35.0 & 3.0 & & - \\
\hline 9603SOLA1 & $\mathrm{ZN}$ & 36.6 & 1.6 & & \\
\hline 9603SOLA1 & $\mathrm{ZN}$ & 39.4 & 1.7 & & \\
\hline 9603SOLA1 & $\mathrm{ZN}$ & 39.8 & 9.3 & & \\
\hline $9603 \mathrm{SOLH} 1$ & ZN & 38.0 & 0.0 & & \\
\hline 9603SONA1 & $\mathrm{ZN}$ & 45.0 & 0.0 & & \\
\hline 9603SONL1 & $\mathrm{ZN}$ & 30.2 & 0.0 & & \\
\hline $96035 O O B 1$ & $\mathrm{ZN}$ & 33.0 & 0.0 & & \\
\hline 9603SOOR1 & $\mathrm{ZN}$ & 41.0 & 0.5 & & \\
\hline $9603 \mathrm{SOSC} 1$ & $\mathrm{ZN}$ & 35.2 & 0.0 & & \\
\hline 9603SOSW1 & $\mathrm{ZN}$ & 33.9 & 0.0 & & \\
\hline 9603SOSW1 & $\mathrm{ZN}$ & 33.8 & 0.0 & & \\
\hline 9603SOSW1 & $\mathrm{ZN}$ & 34.7 & 0.0 & & \\
\hline 9603SOUC1 & $\mathrm{ZN}$ & 42.5 & 4.3 & & \\
\hline 9603SOUP1 & $\mathrm{ZN}$ & 38.1 & 0.0 & & \\
\hline
\end{tabular}

Records printed: 25

$\begin{array}{lc}\text { Statistical Summary } \\ \text { (excluding outliers as determined by Kolmogorov-Smirnov Test) } \\ \text { Mean } & 36.0 \\ \text { Standard Deviation } & 4.4 \\ \text { Median } & 35.0 \\ \text { 15 percentile } & 33.0 \\ 85 \text { percentile } & 40.7 \\ \text { N } & 25\end{array}$

EML results were determined using SW846 method 3050 hotplate digestion with ICP or graphite furnace AA determination. The samples did not appear to totally digest using this method so closed vessel microwave digestion, method 3051 , was used resulting in higher analyte recovery. 


\section{Participating Laboratories in EML QAP44}

\section{Laboratories Reporting Data}

\section{Code Laboratory Name}

AG Paragon Analytics, Inc. , Fort Collins, CO

AR Accu-Labs Research Inc., Golden, CO

CH California State Dept. Health Serv. Sanitation \& Radiation Laborat, Berkeley, CA

DC Datachem Laboratories, Salt Lake City, UT

ES Environmental Sci. \& Engr., Inc. Inorganic Analyt.Chem., Gainesville, FL

FG FGL Environmental, Santa Paula, CA

GA Lockheed Martin Rm. 333 Bldg X710, Pikton, OH

HL Heritage Laboratories, Inc. , Romeoville, IL

IS Quanterra-St. Louis , Earth City, MO

JP Japan Chemical Analysis Center 295-3 Sanno-Cho, Chiba 263, Japan

LA Analytical Services, CST-3 MS K484, Los Alamos, NM

LH Lockheed Analytical Laboratory, Las Vegas, NV

NA USEPA NAREL, Montgomery, AL

NL FERMCO BLDG 15, room C-11, Cincinnati, OH

OB OBG Laboratories, East Syracuse, NY

OR Oak Ridge National Lab Bldg 4500-N Rm G-5, Oak Ridge, TN

SC S-3 Division Maxwell Labs, La Jolla, CA

SW Southwest Research Institute, Div. 01 P.0. Drawer 28510, San Antonio, TX

UC Lockheed Martin RM 60 BLDG C-710, Paducah, KY

UP Y-12 Plant, ASO, OC Laboratory 113C Union Valley Road, Oak Ridge, TN

Total Reporting Labs: 20 


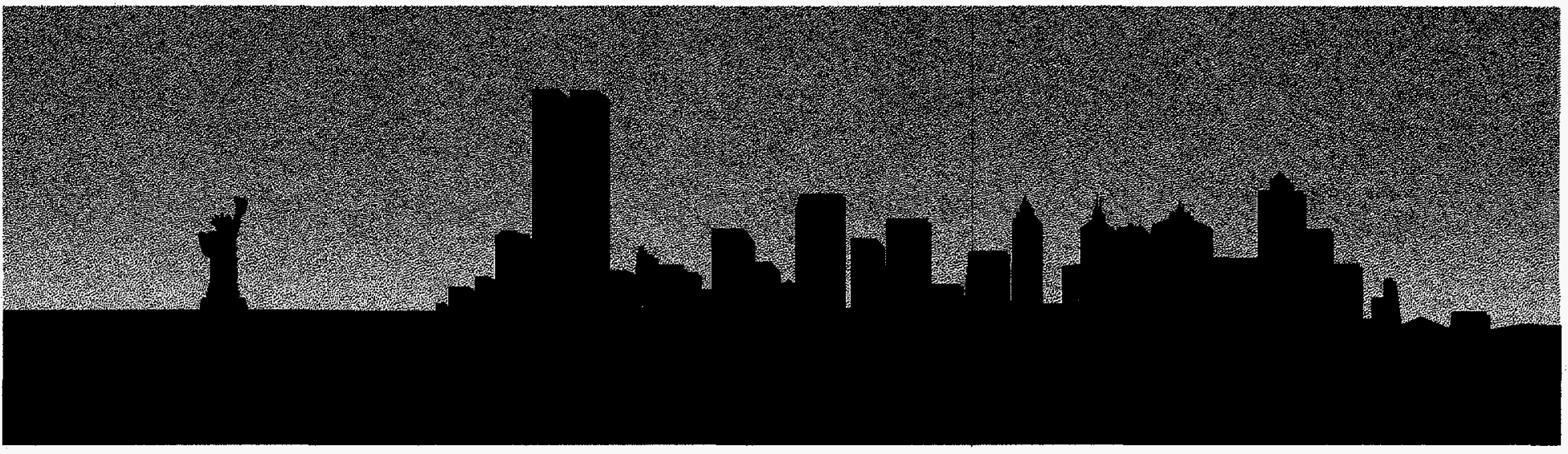

